\title{
Dynamic Pricing and Production Control of an Inventory System with Remanufacturing
}

\author{
Chunyan Gao, ${ }^{1}$ Yao Wang, ${ }^{2}$ Liang Xu, ${ }^{2}$ and Yi Liao ${ }^{2}$ \\ ${ }^{1}$ Department of Management Science, Southwestern University of Finance and Economics, Chengdu, Sichuan 611130, China \\ ${ }^{2}$ School of Business Administration, Southwestern University of Finance and Economics, Chengdu, Sichuan 611130, China \\ Correspondence should be addressed to Chunyan Gao; gaochy_2007@126.com
}

Received 14 September 2015; Revised 14 November 2015; Accepted 24 November 2015

Academic Editor: Young Hae Lee

Copyright (c) 2015 Chunyan Gao et al. This is an open access article distributed under the Creative Commons Attribution License, which permits unrestricted use, distribution, and reproduction in any medium, provided the original work is properly cited.

\begin{abstract}
We consider optimal pricing and manufacturing control of a continuous-review inventory system with remanufacturing. Customer demand and product return follow independent Poisson processes. Customer demand is filled by serviceable product, which can be either manufactured or remanufactured from the returned product. The lead times for both manufacturing and remanufacturing are exponentially distributed. The objective is to maximize the expected total discounted profit over an infinite planning horizon. We characterize the structural properties of the optimal policy through the optimality equation. Specifically, the optimal manufacturing policy is a base-stock policy with the base-stock level nonincreasing in the return inventory level. The optimal pricing policy is also a threshold policy, where the threshold level is nonincreasing in the return inventory level.
\end{abstract}

\section{Introduction}

With increasing awareness of sustainable development, and more stringent government regulations, companies are gradually switching focus from profits to triple bottom lines (profit, people, and planet) in their business operations. Remanufacturing, which converts used products into like-new ones, has gained momentum recently as it not only reduces waste and consumption of natural resources but also produces companies economic benefit by reducing production costs and increasing service levels. Meanwhile, governments in Europe and North America have established legislation to urge manufacturers to accept used products and carry out remanufacturing. Examples of remanufacturing programs include those of Caterpillar, IBM, Kodak, and Fuji-Xerox.

One operational challenge in remanufacturing is production planning and pricing management because of uncertain returned product numbers and customer demand volatility. In this paper, we consider optimal pricing and production control of a continuous-review, infinite-horizon inventory system with remanufacturing. Serviceable products that fill customer demand can be either manufactured or remanufactured from the returned products (also called "core").
The system keeps both serviceable product inventory and core inventory. Demand that cannot be satisfied by available serviceable inventory is backlogged. Lead times for both manufacturing and remanufacturing are exponentially distributed. The objective is to maximize the expected total discounted profit over an infinite planning horizon. We characterize the optimal policy through the optimality equation. Specifically, the optimal manufacturing policy follows a basestock policy with the base-stock level nonincreasing in the core inventory level. The optimal pricing policy is also a threshold policy with threshold level nonincreasing in the return inventory level.

Growth of the remanufacturing industry has stimulated extensive research in the field. Ferrer and Whybark [1], Guide [2], and Larson et al. [3] show that remanufacturing is environmentally friendly and economical. McConocha and Speh [4] note that firms benefit from remanufacturing through reduced production cost, reduced pollution, shortened production lead time, new market, and improved reputation. Our paper is closely related to prior studies on production planning and inventory control of remanufacturing systems. The related work can be divided into two streams: the periodic-review systems and the continuous-review systems. 
Regarding periodic-review systems, Simpson [5] considers a system with zero remanufacturing and manufacturing lead times. The optimal control policy is shown to be characterized by constant thresholds. Inderfurth [6] subsequently extends Simpson's [5] work to models with positive lead times and shows that with equal and deterministic remanufacturing and manufacturing processing times, the optimal policy is the same as the zero lead time model. Zhou et al. [7] study a similar remanufacturing system but with multiple types of remanufacturable products. They derive that the optimal remanufacturing-manufacturing-disposal policy is characterized by a sequence of constant parameters. Tao et al. [8] further consider random yield in remanufacturing inventory systems. DeCroix and Zipkin [9] and DeCroix [10] study multiechelon inventory systems with returns. Our paper differs from such works in that we consider a continuousreview system rather than a periodic-review system. Furthermore, the literature on the periodic-review systems almost assumed that the lead times of the remanufacturing process and the manufacturing process are equal and deterministic. But our paper assumes that the remanufacturing process and the manufacturing process are stochastic and could not necessarily be equal.

Papers on continuous-review models mainly focus on performance evaluation. Heyman [11] and Muckstadt and Isacc [12] formulate the remanufacturing systems as queueing systems. Using the analysis of quasi-birth-and-death process, they obtain the stationary distributions of the systems. Van der Laan and Salomon [13] and Van der Laan et al. [14] consider the push and pull systems. DeCroix et al. [15] study an assemble-to-order system with return; they provide an algorithm to calculate the near-optimal base-stock level. Our paper differs from the previous literature in that we focus on exploring the structural properties of the optimal control policy rather than the performance evaluation. Kim et al. [16] consider a remanufacturing system with all the processes exponentially distributed. The final product is sold at a fixed price. They obtain the structural properties of the optimal policy, including optimal manufacturing, remanufacturing, and disposal policies. Our paper differs from their work in that we assume that the price of the final product is controllable. It could be sold at either high or low price based on the serviceable product inventory. We focus on exploring the optimal pricing and manufacturing strategy.

The remainder of this paper is organized as follows. We specify the model details in Section 2. In Section 3, we examine the structural properties of the value function and characterize the optimal control policies, concluding with a discussion on possible extensions in Section 4.

\section{The Model}

Consider a firm managing a hybrid inventory system with remanufacturing. The serviceable product that fills customer demand is either manufactured from new parts or remanufactured from returned products (also called core). We assume that customers feel indifferent whether a serviceable product is manufactured or remanufactured. This happens in remanufacturing of refillable containers, such as printer cartridges and single-use cameras. Return arrives randomly and follows a Poisson process with rate $r$. We assume that return process is independent of the demand arriving process. This assumption is widely used in the previous literature; see Kim et al. [16] and references therein. The returns enter the system and are stocked at the core inventory waiting for remanufacturing. A cost $h_{s}$ is incurred for holding one unit of core per unit time. The remanufacturing and manufacturing lead times are exponentially distributed with rates $\mu_{r}$ and $\mu_{m}$, respectively. Let $c_{r}$ and $c_{m}$ denote the unit remanufacturing and manufacturing cost with $c_{m} \geq c_{r}$, which means that the remanufacturing is more cost-efficient. A completed product from remanufacturing or manufacturing process is stocked at the serviceable inventory and incurs a holding cost $h_{m}$ per unit per unit time. The demand arrives according to a nonhomogeneous Poisson process with price-dependent arrival rate at that time. There are two sale prices: high price $p_{1}$ and low price $p_{2}$, with corresponding arrival rates $\lambda_{1}$ and $\lambda_{2}$. To make low price economically reasonable, we assume that $p_{1}>p_{2}$ and $\lambda_{1}<\lambda_{2}$. Each customer requires only one unit of the serviceable product. The demand that cannot be satisfied immediately from the on-hand serviceable inventory is backlogged and incurs a backorder cost $b$ per unit per unit time. Let

$$
\xi=\frac{\lambda_{2} p_{2}-\lambda_{1} p_{1}}{\lambda_{2}-\lambda_{1}},
$$

which is defined as the marginal profit by changing the price from high to low. Furthermore, we require that $\xi<b / \beta$, which means that the marginal loss through raising price is smaller than the net value of backlogging an order, and thus it has incentive to rase the price.

The firm controls both the manufacturing process and price with an aim to minimize the expected total discounted cost over an infinite planning horizon. Specifically, the manufacturing control characterizes whether to manufacture; the price decision controls the sale price of the serviceable product by observing the current inventory level. The model is illustrated in Figure 1.

Let $(X(t), Y(t))$ be the system state, where $X(t)$ denotes the serviceable inventory level at time $t$ and $Y(t)$ denotes the core inventory level at time $t$. Then the state space $\Omega, \Omega=$ $\left\{(x, y): x \in Z, y \in Z^{+}\right\}$. The expected discounted total profit over an infinite planning horizon under a policy $\pi$ with starting state $(x, y)$ can be calculated as

$$
\begin{aligned}
& V^{\pi}(x, y)=E\left\{\int _ { 0 } ^ { + \infty } e ^ { - \beta t } \left\{p(t) d N^{\pi}(t)-c_{m} d P_{m}^{\pi}(t)\right.\right. \\
& \left.\left.-c_{r} d P_{r}^{\pi}(t)-G(X(t), Y(t)) d t\right\}\right\},
\end{aligned}
$$

where $0 \leq \beta<1$ is the discount factor, $p(t)$ denotes the price listed at time $t, N^{\pi}(t)$ denotes the total demands up to time $t$, $P_{m}^{\pi}(t)$ and $P_{r}^{\pi}(t)$ denote the total numbers of manufacturing and remanufacturing products up to time $t$, respectively; and $G(X(t), Y(t))$ denotes the sum of the holding cost of core products and serviceable products and backorder cost; that is, $G(X(t), Y(t))=h_{r} Y(t)+h_{s}[X(t)]^{+}-b[X(t)]^{-}$. 


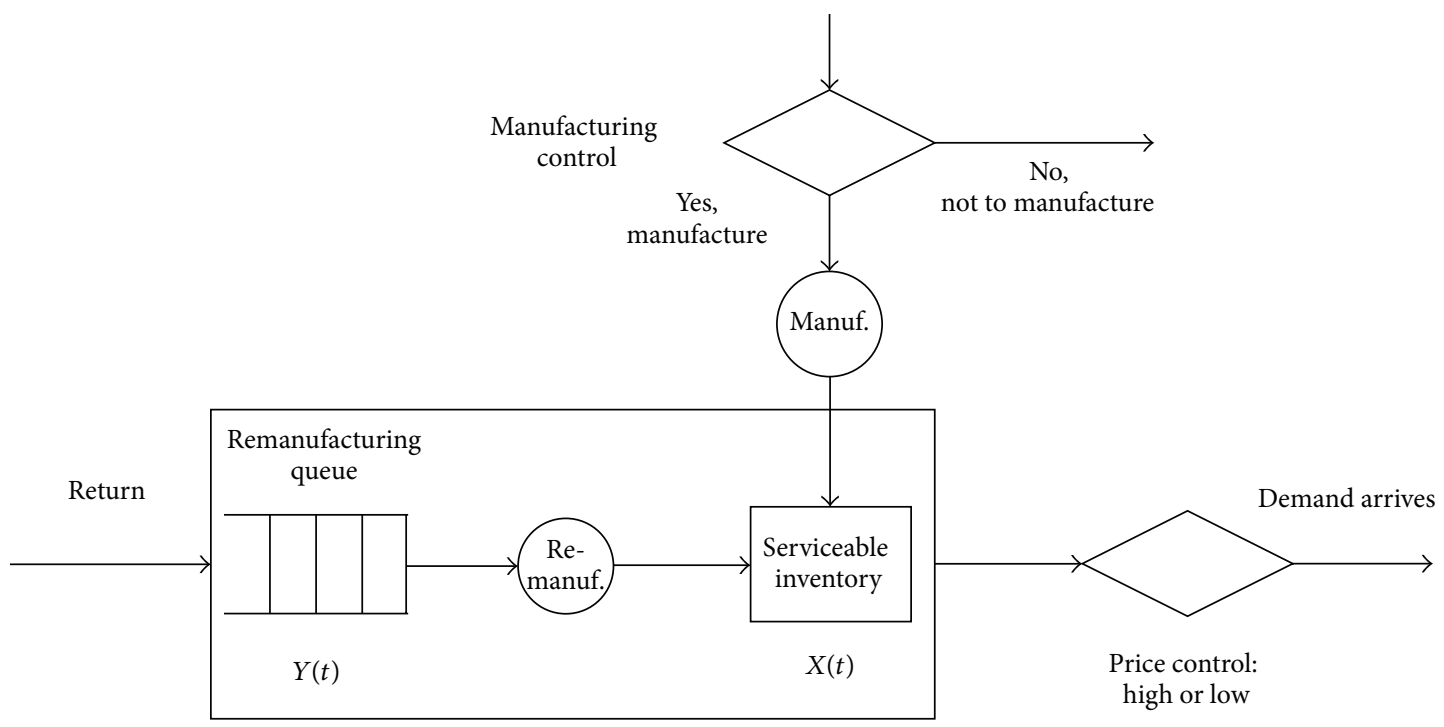

FIGURE 1: The remanufacturing inventory system.

A policy $\pi^{*}$ is optimal if it satisfies

$$
V^{\pi^{*}}(x, y)=\sup _{\pi} V^{\pi}(x, y) .
$$

To facilitate notation, we omit the superscript from $V^{\pi^{*}}(x, y)$ to denote the optimal expected discounted total profit. Moreover, throughout the paper, we use "increasing" and "decreasing" in a nonstrict sense, that is, they mean "nondecreasing" and "nonincreasing," respectively.

\section{Optimal Analysis}

In this section, we aim to find the optimal pricing and manufacturing policies that minimize the long run expected discounted total cost. The exponential distributions of all processes facilitate our analysis. We formulate the system as a Markov decision process. Following Lippman [17], we rescale the time unit and let $\beta+\lambda_{1}+\lambda_{2}+r+\mu_{m}+\mu_{r}=1$. Rewriting (2), we get the optimality equation given by

$$
\begin{aligned}
V(x, y)= & \mathbb{} V(x, y) \\
= & -G(x, y)+r V(x, y+1)+\mu_{r} \mathbb{\mathbb { T }}_{r} V(x, y) \\
& +\mu_{m} \mathbb{\mathbb { T }}_{m} V(x, y)+\mathbb{\mathbb { T }}_{p} V(x, y),
\end{aligned}
$$

where $\mathbb{T}, \mathbb{T}_{m}, \mathbb{T}_{p}$ and $\mathbb{T}_{r}$ are operators; $\mathbb{T}_{m} V(x, y)=\max \{V(x+$ $\left.1, y)-c_{m}, V(x, y)\right\}, \mathbb{T}_{p} V(x, y)=\max \left\{\lambda_{1}\left[p_{1}+V(x-1, y)\right]+\right.$ $\left.\lambda_{2} V(x, y), \lambda_{1} V(x, y)+\lambda_{2}\left[p_{2}+V(x-1, y)\right]\right\}$, and

$$
\mathbb{T}_{r} V(x, y)= \begin{cases}V(x+1, y-1)-c_{r}, & y>0 \\ V(x, y), & y=0 .\end{cases}
$$

In (4), $G(x, y)$ is the inventory holding cost and backorder cost; the remaining terms denote the expected discounted total profit from next epoch, at which system state changes.
Operator $\mathbb{T}_{p}$ determines the sale price, high or low, while $\mathbb{T}_{m}$ determines whether or not to manufacture. Note that the firm can remanufacture only when there are cores available; that is, $y>0$. The following lemma follows directly from the optimality equation (4).

Lemma 1. (1) It is optimal to charge a high price in state $(x, y)$ if $V(x, y)-V(x-1, y) \geq \xi$; otherwise charge a low price.

(2) It is optimal to manufacture in state $(x, y)$ if $V(x+1$, $y)-V(x, y) \geq c_{m}$; otherwise do not manufacture.

Proof. (1) From operator $\mathbb{T}_{p}$, we get that if $\lambda_{1}\left[p_{1}+V(x-1, y)\right]+$ $\lambda_{2} V(x, y) \geq \lambda_{1} V(x, y)+\lambda_{2}\left[p_{2}+V(x-1, y)\right]$, that is, $V(x, y)-$ $V(x-1, y) \geq \xi$, then it is optimal to charge high price $p_{1}$; otherwise charge a low price.

(2) From operator $\mathbb{T}_{m}$, we can easily get (2).

To facilitate the analysis of the structural properties of the optimal control policy, we define a function set $\mathscr{V}$ on the state space $\Omega$; if a function $V(x, y) \in \mathscr{V}$, then it satisfies the following properties:

P1 (concavity). Consider

$$
V(x+2, y)-V(x+1, y) \leq V(x+1, y)-V(x, y)
$$

P2 (submodular). Consider

$$
\begin{gathered}
V(x+1, y+1)-V(x, y+1) \\
\leq V(x+1, y)-V(x, y)
\end{gathered}
$$

P3. Consider

$$
\begin{aligned}
& V(x+2, y)-V(x+1, y) \\
& \quad \leq V(x+1, y+1)-V(x, y+1) .
\end{aligned}
$$

Lemma 2. If $V(x, y) \in \mathscr{V}$, then $\mathbb{} V(x, y) \in \mathscr{V}$. 
Proof. For notational convenience, we introduce the following difference operators $\mathbb{D}$, where $\mathbb{D}\{V(x, y)\}=V(x+1, y)$ $V(x, y)$. It is easy to verify that the first two terms in (4) satisfy P1-P3. Therefore we only have to prove that operators $\mathbb{T}_{r}, \mathbb{T}_{m}$, and $\mathbb{T}_{p}$ preserve properties $\mathrm{P} 1-\mathrm{P} 3$.

$\mathbb{T}_{r}$ Preserves Properties P1-P3. It is easy to show that $\mathbb{T}_{r}$ preserves $\mathrm{P} 1-\mathrm{P} 3$ when $y \neq 0$; therefore we only have to prove that $\mathbb{T}_{r}$ preserves $\mathrm{P} 1-\mathrm{P} 3$ when $y=0$.

P1. Consider

$$
\begin{aligned}
\mathbb{D}\left\{\mathbb{\mathbb { T }}_{r} V(x+1,0)\right\} & =V(x+2,0)-V(x+1,0) \\
& \leq V(x+1,0)-V(x, 0) \\
& =\mathbb{D}\left\{\mathbb{\mathbb { T }}_{r} V(x, 0)\right\},
\end{aligned}
$$

which is due to $\mathrm{P} 1$.

P2. Consider

$$
\begin{aligned}
\mathbb{D}\left\{\mathbb{T}_{r} V(x, 1)\right\}= & {\left[V(x+2,0)-c_{r}\right] } \\
& -\left[V(x+1,0)-c_{r}\right] \\
\leq & V(x+1,0)-V(x, 0) \\
\leq & \mathbb{D}\left\{\mathbb{T}_{r} V(x, 0)\right\}
\end{aligned}
$$

due to P1.

P3. Consider

$$
\begin{aligned}
\mathbb{D}\left\{\mathbb{T}_{r} V(x+1,0)\right\}= & V(x+2,0)-V(x+1,0) \\
= & {\left[V(x+2,0)-c_{r}\right] } \\
& -\left[V(x+1,0)-c_{r}\right] \\
\leq & \mathbb{D}\left\{\mathbb{\mathbb { T }}_{r} V(x, 1)\right\} .
\end{aligned}
$$

\section{$\mathbb{T}_{m}$ Preserves Properties $P 1-P 3$}

P1. We have the following three cases.

Case $1\left(c_{m} \leq \mathbb{D}\{V(x+2, y)\} \leq \mathbb{D}\{V(x, y)\}\right)$. Consider

$$
\begin{aligned}
\mathbb{D}\left\{\mathbb{T}_{m} V(x+1, y)\right\} \leq & {\left[V(x+3, y)-c_{m}\right] } \\
& -\left[V(x+2, y)-c_{m}\right] \\
\leq & {\left[V(x+2, y)-c_{m}\right] } \\
& -\left[V(x+1, y)-c_{m}\right] \\
\leq & \mathbb{D}\left\{\mathbb{T}_{m} V(x, y)\right\},
\end{aligned}
$$

which is due to $\mathrm{P} 1$.
Case $2\left(\mathbb{D}\{V(x+2, y)\} \leq c_{m} \leq \mathbb{D}\{V(x, y)\}\right)$. Consider

$$
\begin{aligned}
\mathbb{D}\left\{\mathbb{T}_{m} V(x+1, y)\right\} \leq & V(x+2, y)-V(x+1, y) \\
= & {\left[V(x+2, y)-c_{m}\right] } \\
& -\left[V(x+1, y)-c_{m}\right] \\
\leq & \mathbb{D}\left\{\mathbb{T}_{m} V(x, y)\right\} .
\end{aligned}
$$

Case $3\left(\mathbb{D}\{V(x+2, y)\} \leq \mathbb{D}\{V(x, y)\} \leq c_{m}\right)$. Consider

$$
\begin{aligned}
\mathbb{D}\left\{\mathbb{T}_{m} V(x+1, y)\right\} & \leq V(x+2, y)-V(x+1, y) \\
& \leq V(x+1, y)-V(x, y) \\
& \leq \mathbb{D}\left\{\mathbb{T}_{m} V(x, y)\right\},
\end{aligned}
$$

which is due to P1.

P2. Considering $\mathbb{D}\{V(x+1, y+1)\} \leq \mathbb{D}\{V(x+1, y)\} \leq$ $\mathbb{D}\{V(x, y)\}$ due to $\mathrm{P} 1$ and $\mathrm{P} 2$, we have the following three cases.

Case $1\left(c_{m} \leq \mathbb{D}\{V(x+1, y+1)\} \leq \mathbb{D}\{V(x, y)\}\right)$. Consider

$$
\begin{aligned}
\mathbb{D}\left\{\mathbb{T}_{m} V(x, y+1)\right\} \leq & {\left[V(x+2, y+1)-c_{m}\right] } \\
& -\left[V(x+1, y+1)-c_{m}\right] \\
\leq & {\left[V(x+2, y)-c_{m}\right] } \\
& -\left[V(x+1, y)-c_{m}\right] \\
\leq & \mathbb{D}\left\{\mathbb{T}_{m} V(x, y)\right\},
\end{aligned}
$$

which is due to $\mathrm{P} 2$.

Case $2\left(\mathbb{D}\{V(x+1, y+1)\} \leq c_{m} \leq \mathbb{D}\{V(x, y)\}\right)$. Consider

$$
\begin{aligned}
\mathbb{D}\left\{\mathbb{T}_{m} V(x, y+1)\right\} \leq & V(x+1, y+1) \\
& -\left[V(x+1, y+1)-c_{m}\right] \\
= & V(x+1, y) \\
& -\left[V(x+1, y)+c_{m}\right] \\
\leq & \mathbb{D}\left\{\mathbb{T}_{m} V(x, y)\right\},
\end{aligned}
$$

which is due to $\mathrm{P} 1$ and $\mathrm{P} 2$.

Case $3\left(\mathbb{D}\{V(x+1, y+1)\} \leq \mathbb{D}\{V(x, y)\} \leq c_{m}\right)$. Consider

$$
\begin{aligned}
\mathbb{D}\left\{\mathbb{T}_{m} V(x, y+1)\right\} & \leq V(x+1, y+1)-V(x, y+1) \\
& \leq V(x+1, y)-V(x, y) \\
& \leq \mathbb{D}\left\{\mathbb{T}_{m} V(x, y)\right\},
\end{aligned}
$$

which is due to $\mathrm{P} 2$.

P3. Considering $\mathbb{D}\{V(x+2, y)\} \leq \mathbb{D}\{V(x+1, y+1)\} \leq$ $\mathbb{D}\{V(x, y+1)\}$, which is due to $\mathrm{P} 2$, we have following three cases. 
Case $1\left(c_{m} \leq \mathbb{D}\{V(x+2, y)\} \leq \mathbb{D}\{V(x, y+1)\}\right)$. Consider

$$
\begin{aligned}
\mathbb{D}\left\{\mathbb{\mathbb { T }}_{m} V(x+1, y)\right\} \leq & {\left[V(x+3, y)-c_{m}\right] } \\
& -\left[V(x+2, y)-c_{m}\right] \\
\leq & {\left[V(x+2, y+1)-c_{m}\right] } \\
& -\left[V(x+1, y+1)-c_{m}\right] \\
\leq & \mathbb{D}\left\{\mathbb{T}_{m} V(x, y+1)\right\},
\end{aligned}
$$

which is due to $\mathrm{P} 3$.

Case $2\left(\mathbb{D}\{V(x+2, y)\} \leq c_{m} \leq \mathbb{D}\{V(x, y+1)\}\right)$. Consider

$$
\begin{aligned}
\mathbb{D}\left\{\mathbb{\mathbb { T }}_{m} V(x+1, y)\right\} \leq & V(x+2, y) \\
& -\left[V(x+2, y)-c_{m}\right] \\
= & V(x+1, y+1) \\
& -\left[V(x+1, y+1)-c_{m}\right] \\
\leq & \mathbb{D}\left\{\mathbb{T}_{m} V(x, y+1)\right\},
\end{aligned}
$$

which is due to $\mathrm{P} 1$ and $\mathrm{P} 3$.

Case $3\left(\mathbb{D}\{V(x+2, y)\} \leq \mathbb{D}\{V(x, y+1)\} \leq c_{m}\right)$. Consider

$$
\begin{aligned}
\mathbb{D}\left\{\mathbb{T}_{m} V(x+1, y)\right\} & \leq V(x+2, y)-V(x+1, y) \\
& \leq V(x+1, y+1)-V(x, y+1) \\
& \leq \mathbb{D}\left\{\mathbb{T}_{m} V(x, y+1)\right\},
\end{aligned}
$$

which is due to $\mathrm{P} 3$.

\section{$\mathbb{T}_{p}$ Preserves Properties P1-P3}

P1. Considering $\mathbb{D}\{V(x+1, y)\} \leq \mathbb{D}\{V(x-1, y)\}$ due to $\mathrm{P} 1$, we have the following three cases.

Case $1(\xi \leq \mathbb{D}\{V(x+1, y)\} \leq \mathbb{D}\{V(x-1, y)\})$. Consider

$$
\begin{aligned}
& \mathbb{D}\left\{\mathbb{\pi}_{p} V(x+1, y)\right\} \\
& \leq\left\{\lambda_{1}\left[p_{1}+V(x+1, y)\right]+\lambda_{2} V(x+2, y)\right\} \\
& -\left\{\lambda_{1}\left[p_{1}+V(x, y)\right]+\lambda_{2} V(x+1, y)\right\} \\
& \leq\left\{\lambda_{1}\left[p_{1}+V(x, y)\right]+\lambda_{2} V(x+1, y)\right\} \\
& -\left\{\lambda_{1}\left[p_{1}+V(x-1, y)\right]+\lambda_{2} V(x, y)\right\} \\
& \leq \mathbb{D}\left\{\mathbb{T}_{p} V(x, y)\right\},
\end{aligned}
$$

where the inequality is due to P1.
Case $2(\mathbb{D}\{V(x+1, y)\} \leq \xi \leq \mathbb{D}\{V(x-1, y)\})$. Consider

$$
\begin{aligned}
\mathbb{D}\{ & \left.\mathbb{T}_{p} V(x+1, y)\right\} \\
\leq & \left\{\lambda_{1} V(x+2, y)+\lambda_{2}\left[p_{2}+V(x+1, y)\right]\right\} \\
& -\left\{\lambda_{1} V(x+1, y)+\lambda_{2}\left[p_{2}+V(x, y)\right]\right\} \\
\leq & \left\{\lambda_{1}\left[p_{1}+V(x, y)\right]+\lambda_{2} V(x+1, y)\right\} \\
& -\left\{\lambda_{1}\left[p_{1}+V(x-1, y)\right]+\lambda_{2} V(x, y)\right\} \\
\leq & \mathbb{D}\left\{\mathbb{T}_{p} V(x, y)\right\},
\end{aligned}
$$

where the inequality is due to P1.

Case $3(\mathbb{D}\{V(x+1, y)\} \leq \mathbb{D}\{V(x-1, y)\} \leq \xi)$. Consider

$$
\begin{aligned}
\mathbb{D}\{ & \left.\mathbb{T}_{p} V(x+1, y)\right\} \\
\leq & \left\{\lambda_{1} V(x+2, y)+\lambda_{2}\left[p_{2}+V(x+1, y)\right]\right\} \\
& -\left\{\lambda_{1} V(x+1, y)+\lambda_{2}\left[p_{2}+V(x, y)\right]\right\} \\
\leq & \left\{\lambda_{1} V(x+1, y)+\lambda_{2}\left[p_{2}+V(x, y)\right]\right\} \\
& -\left\{\lambda_{1} V(x, y)+\lambda_{2}\left[p_{2}+V(x-1, y)\right]\right\} \\
\leq & \mathbb{D}\left\{\mathbb{T}_{p} V(x, y)\right\},
\end{aligned}
$$

where the inequality is due to P1.

P2. Considering $\mathbb{D}\{V(x, y+1)\} \leq \mathbb{D}\{V(x, y)\} \leq \mathbb{D}\{V(x-1, y)\}$ due to $\mathrm{P} 3$ and $\mathrm{P} 1$, we have the following three cases.

Case $1(\xi \leq \mathbb{D}\{V(x, y+1)\} \leq \mathbb{D}\{V(x-1, y)\})$. Consider

$$
\begin{aligned}
\mathbb{D}\{ & \left.\mathbb{T}_{p} V(x, y+1)\right\} \\
\leq & \left\{\lambda_{1}\left[p_{1}+V(x, y+1)\right]+\lambda_{2} V(x+1, y+1)\right\} \\
& -\left\{\lambda_{1}\left[p_{1}+V(x-1, y+1)\right]+\lambda_{2} V(x, y+1)\right\} \\
\leq & \left\{\lambda_{1}\left[p_{1}+V(x, y)\right]+\lambda_{2} V(x+1, y)\right\} \\
& -\left\{\lambda_{1}\left[p_{1}+V(x-1, y)\right]+\lambda_{2} V(x, y)\right\} \\
\leq & \mathbb{D}\left\{\mathbb{T}_{p} V(x, y)\right\},
\end{aligned}
$$

where the inequality is due to $\mathrm{P} 2$.

Case $2(\mathbb{D}\{V(x, y+1)\} \leq \xi \leq \mathbb{D}\{V(x-1, y)\})$. Consider

$$
\begin{aligned}
\mathbb{D}\{ & \left.\mathbb{\mathbb { T }}_{p} V(x, y+1)\right\} \\
\leq & \left\{\lambda_{1} V(x+1, y+1)+\lambda_{2}\left[p_{2}+V(x, y+1)\right]\right\} \\
& -\left\{\lambda_{1}\left[p_{1}+V(x-1, y+1)\right]+\lambda_{2} V(x, y+1)\right\} \\
\leq & \left\{\lambda_{1} V(x+1, y)+\lambda_{2}\left[p_{2}+V(x, y)\right]\right\} \\
& -\left\{\lambda_{1}\left[p_{1}+V(x-1, y)\right]+\lambda_{2} V(x, y)\right\} \\
\leq & \mathbb{D}\left\{\mathbb{\mathbb { T }}_{p} V(x, y)\right\},
\end{aligned}
$$

where the inequality is due to $\mathrm{P} 2$. 
Case $3(\mathbb{D}\{V(x, y+1)\} \leq \mathbb{D}\{V(x-1, y)\} \leq \xi)$. Consider

$$
\begin{aligned}
\mathbb{D}\{ & \left.\mathbb{\mathbb { V }}_{p} V(x+1, y)\right\} \\
\leq & \left\{\lambda_{1} V(x+1, y+1)+\lambda_{2}\left[p_{2}+V(x, y+1)\right]\right\} \\
& -\left\{\lambda_{1} V(x, y+1)+\lambda_{2}\left[p_{2}+V(x-1, y+1)\right]\right\} \\
\leq & \left\{\lambda_{1} V(x+1, y)+\lambda_{2}\left[p_{2}+V(x, y)\right]\right\} \\
& -\left\{\lambda_{1} V(x, y)+\lambda_{2}\left[p_{2}+V(x-1, y)\right]\right\} \\
\leq & \mathbb{D}\left\{\mathbb{T}_{p} V(x, y)\right\},
\end{aligned}
$$

where the inequality is due to $\mathrm{P} 2$.

P3. Considering $\mathbb{D}\{V(x, y+1)\} \leq D V(x, y+1) \leq \mathbb{D}\{V(x-$ $1, y+1)\}$ due to P3 and P1, we have the following three cases.

Case $1(\xi \leq \mathbb{D}\{V(x+1, y)\} \leq \mathbb{D}\{V(x-1, y+1)\})$. Consider

$$
\begin{aligned}
\mathbb{D}\{ & \left.\mathbb{\mathbb { T }}_{p} V(x+1, y)\right\} \\
\leq & \left\{\lambda_{1}\left[p_{1}+V(x+1, y)\right]+\lambda_{2} V(x+2, y)\right\} \\
& -\left\{\lambda_{1}\left[p_{1}+V(x, y)\right]+\lambda_{2} V(x+1, y)\right\} \\
\leq & \left\{\lambda_{1}\left[p_{1}+V(x, y+1)\right]+\lambda_{2} V(x+1, y+1)\right\} \\
& -\left\{\lambda_{1}\left[p_{1}+V(x-1, y+1)\right]+\lambda_{2} V(x, y+1)\right\} \\
\leq & \mathbb{D}\left\{\mathbb{T}_{p} V(x, y)\right\},
\end{aligned}
$$

where the inequality is due to $\mathrm{P} 3$.

Case $2(\mathbb{D}\{V(x+1, y)\} \leq \xi \leq \mathbb{D}\{V(x-1, y+1)\})$. Consider

$$
\begin{aligned}
\mathbb{D}\{ & \left.\mathbb{T}_{p} V(x+1, y)\right\} \\
\leq & \left\{\lambda_{1} V(x+2, y)+\lambda_{2}\left[p_{2}+V(x+1, y)\right]\right\} \\
& -\left\{\lambda_{1}\left[p_{1}+V(x, y)\right]+\lambda_{2} V(x+1, y)\right\} \\
\leq & \left\{\lambda_{1} V(x+1, y+1)+\lambda_{2}\left[p_{2}+V(x, y+1)\right]\right\} \\
& -\left\{\lambda_{1}\left[p_{1}+V(x-1, y+1)\right]+\lambda_{2} V(x, y+1)\right\} \\
\leq & \mathbb{D}\left\{\mathbb{T}_{p} V(x, y)\right\},
\end{aligned}
$$

where the inequality is due to that $V(x+2, y)-V(x+1, y+1) \leq$ $V(x, y)-V(x-1, y+1)$ according to $\mathrm{P} 3$.

Case $3(\mathbb{D}\{V(x+1, y)\} \leq \mathbb{D}\{V(x-1, y+1)\} \leq \xi)$. Consider

$$
\begin{aligned}
\mathbb{D}\{ & \left.\mathbb{T}_{p} V(x+1, y)\right\} \\
\leq & \left\{\lambda_{1} V(x+2, y)+\lambda_{2}\left[p_{2}+V(x+1, y)\right]\right\} \\
& -\left\{\lambda_{1} V(x+1, y)+\lambda_{2}\left[p_{2}+V(x, y)\right]\right\} \\
\leq & \left\{\lambda_{1} V(x+1, y+1)+\lambda_{2}\left[p_{2}+V(x, y+1)\right]\right\} \\
& -\left\{\lambda_{1} V(x, y+1)+\lambda_{2}\left[p_{2}+V(x-1, y+1)\right]\right\} \\
\leq & \mathbb{D}\left\{\mathbb{T}_{p} V(x, y)\right\},
\end{aligned}
$$

where the inequality is due to P3.
The concavity property posits that the marginal profit of serviceable inventory is decreasing. The submodularity of $V(x, y)$ implies that the serviceable inventory and the core inventory are substitutable to each other.

After deriving the structural properties of $V(x, y)$, we can now characterize the optimal policy in the following theorem.

Theorem 3. The optimal control policy is characterized by two state-dependent parameters $\left(s_{m}^{*}(y), s_{p}^{*}(y)\right)$. Specifically, in state $(x, y)$,

(a) it is optimal to manufacture if the serviceable inventory level $x \leq s_{m}^{*}(y)$. Furthermore, $s_{m}^{*}(y+1) \leq s_{m}^{*}(y) \leq$ $s_{m}^{*}(y+1)+1$

(b) the optimal price control policy is the threshold policy, specially set high price if the serviceable inventory level $x \leq s_{p}^{*}(y)$; otherwise set low price. Furthermore, $s_{p}^{*}(y+$ 1) $\leq s_{p}^{*}(y)$.

Proof. The manufacturing base-stock level is defined as $s_{m}^{*}(y)=\max _{x}\left\{x: V(x+1, y)-V(x, y) \geq c_{m}\right\}$. Consider $c_{m} \leq V\left(s_{m}^{*}(y)+1, y\right)-V\left(s_{m}^{*}(y), y\right) \leq V\left(s_{m}^{*}(y)+1, y-1\right)-$ $V\left(s_{m}^{*}(y), y-1\right)$, which is due to the definition of $s_{m}^{*}(y)$ and P2; then we have $s_{m}^{*}(y) \leq s_{m}^{*}(y-1)$. Furthermore, consider $c_{m} \geq$ $V\left(s_{m}^{*}(y)+1, y\right)-V\left(s_{m}^{*}(y), y\right) \leq V\left(s_{m}^{*}(y), y+1\right)-V\left(s_{m}^{*}(y)-\right.$ $1, y+1)$ due to P3; then we have $s_{m}^{*}(y+1) \geq s_{m}^{*}(y)-1$.

The price threshold level is $s_{p}^{*}(y)=\max _{x}\{x: V(x+1, y)-$ $V(x, y) \geq \xi\}$. Consider $\xi \leq V\left(s_{p}^{*}(y)+1, y\right)-V\left(s_{p}^{*}(y), y\right) \leq$ $V\left(s_{p}^{*}(y)+1, y-1\right)-V\left(s_{p}^{*}(y), y-1\right)$, which is due to the definition of $s_{p}^{*}(y)$ and P2; then we have $s_{p}^{*}(y) \leq s_{p}^{*}(y-1)$.

Theorem 3 shows that the optimal control policy is statedependent. If it is optimal to manufacture in state $(x, y+1)$, then it is still optimal to manufacture in state $(x, y)$, but one unit decrease in the core inventory leads to at most one unit increase in the manufacturing base-stock level. This implies that manufacturing control policy depends on the core inventory level. The core inventory and the serviceable inventory are substitutable. The reason is that due to the stochastic of the manufacturing and return processes the core inventory may be restored to the original level during the manufacturing process. It is optimal to set high price when the serviceable inventory level is lower than the threshold level. The threshold level is decreasing at the core inventory level, meaning that if it is optimal to set a high price in state $(x, y)$, it is still optimal to adopt a high price when the core inventory level is lower than $y$.

We further visualize the structure of the optimal policy in Figures 2 and 3 using a numerical example. The parameters are $\beta=0.0001, \lambda_{1}=6, \lambda_{2}=10, p_{1}=50, p_{2}=40, \mu_{m}=$ 15, $\mu_{r}=20, r=15, c_{m}=10, c_{r}=5, h_{r}=1, h_{s}=2$, and $b=$ 30. The optimal manufacturing policy is shown in Figure 2, while the optimal pricing policy is shown in Figure 3. We specify the optimal action in each region.

\section{Conclusions}

We consider a continuous-review inventory system, in which the serviceable product can be either manufactured or 


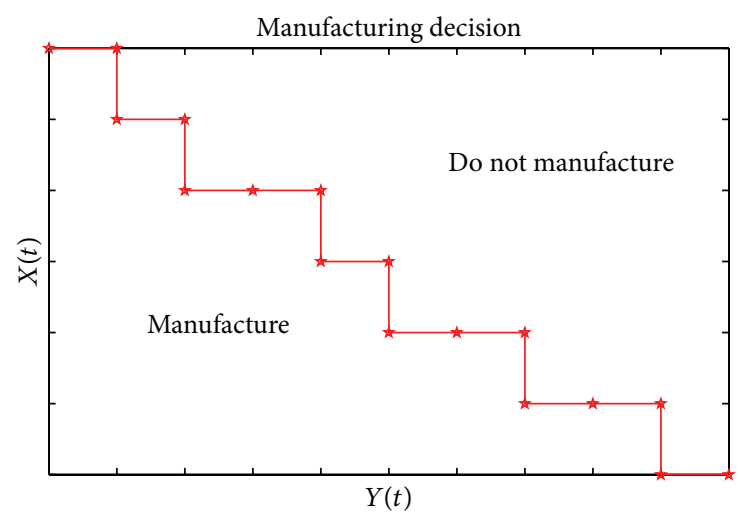

FIGURE 2: The optimal manufacturing policy.

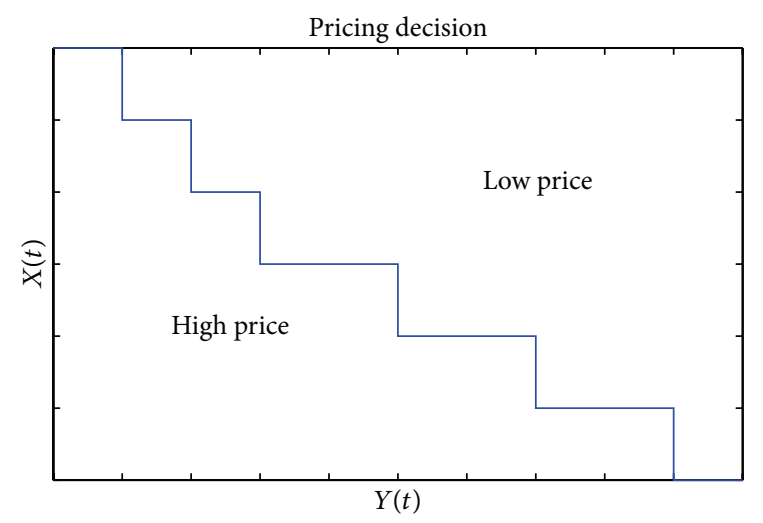

FIgURE 3: The optimal pricing policy.

remanufactured from the returned product. The system keeps both serviceable and returned product inventory. The lead times of manufacturing and remanufacturing are exponentially distributed. We show that the value function preserves properties $\mathrm{P} 1-\mathrm{P} 3$ and characterizes the optimal pricing and manufacturing policies. Different from the previous literature, we consider pricing decision. The optimal control policy of our model depends on the core inventory level. Specifically, the optimal pricing control policy is the threshold-type policy with threshold level nonincreasing in the core inventory level. The optimal manufacturing control policy is the basestock policy with base-stock level nonincreasing in the core inventory.

One possible extension is to consider multiple prices. In this case, the selling price of the serviceable product is chosen among $\left\{p_{k}, k=1, \ldots, n\right\}$ with $p_{1}>p_{2}>\cdots>p_{k}>\cdots>p_{n}$. The associated demand arrival rates are $\left\{\lambda_{k}, k=1, \ldots, n\right\}$ with $\lambda_{1}<\lambda_{2}<\cdots<\lambda_{k}<\cdots<\lambda_{n}$. To facilitate analysis, we further assume that

$$
\begin{aligned}
\xi_{12} & =\frac{\lambda_{2} p_{2}-\lambda_{1} p_{1}}{\lambda_{2}-\lambda_{1}}<\xi_{23}=\frac{\lambda_{3} p_{3}-\lambda_{2} p_{2}}{\lambda_{3}-\lambda_{2}}<\cdots \\
& <\xi_{n-1, n}=\frac{\lambda_{n} p_{n}-\lambda_{n-1} p_{n-1}}{\lambda_{n}-\lambda_{n-1}} .
\end{aligned}
$$

The optimality equation can be formulated as

$$
\begin{aligned}
V(x, y)= & T V(x, y) \\
= & -G(x, y)+r V(x, y+1)+\mu_{r} \mathbb{\mathbb { T }}_{r} V(x, y) \\
& +\mu_{m} \mathbb{\mathbb { T }}_{m} V(x, y)+\mathbb{\mathbb { T }}_{p} V(x, y),
\end{aligned}
$$

where $\mathbb{T}_{p} V(x, y)=\max _{1 \leq k \leq n}\left\{\lambda_{k}\left[p_{k}+V(x-1, y)\right]+\right.$ $\left.\sum_{i \neq k} \lambda_{i} V(x, y)\right\}$.

We can again show that $V(x, y)$ preserves properties P1P3 and characterizes the optimal control policy. For brevity, we ignore the details here. The optimal manufacturing policy possesses a similar structure as the two price cases, while the optimal pricing control policy is given in the following theorem.

Theorem 4. The optimal pricing control is characterized by $n$ regions as follows: there exist $n$ price threshold levels; $s_{p k}^{*}(y)=$ $\max _{x}\left\{x: V(x+1, y)-V(x, y) \geq \xi_{k, k+1}\right\}, k=1,2, \ldots, n-1$.

There are other research directions worth exploring. First, we can study models with correlated demand and product return processes. This will increase the state space and make the analysis more challenging. Second, the product return is not controllable in our model. We can incorporate and examine the return product acquisition effort decision. Finally, the present model assumes that the remanufactured and manufactured products are the same to customers. But, in some cases, the remanufactured and manufactured products are treated as two different products and sold at different prices. The firm can expand the market share by selling the remanufactured products at a lower price in the low-end market, even though the remanufactured products perform exactly the same function as the new products. In this case, we need to model customer demand by considering how customers choose between these two products. And pricing decisions can be incorporated.

\section{Conflict of Interests}

The authors declare that there is no conflict of interests regarding the publication of this paper.

\section{Acknowledgment}

This work was supported in part by the National Natural Science Foundation of China under Grant nos. 71201128, 71201127, and 71302187.

\section{References}

[1] G. Ferrer and D. C. Whybark, "Successful remanufacturing systems and skills," Business Horizons, vol. 43, no. 6, pp. 55-64, 2000.

[2] V. D. R. Guide Jr., "Production planning and control for remanufacturing: industry practice and research needs," Journal of Operations Management, vol. 18, no. 4, pp. 467-483, 2000. 
[3] L. Larson, E. O. Teisbeg, and R. R. Johnson, "Sustainable business: opportunity and value creation," Interfaces, vol. 30, no. 3 , pp. 1-12, 2000.

[4] D. M. McConocha and T. W. Speh, "Remarketing: commercialization of remanufacturing technology," Journal of Business \& Industrial Marketing, vol. 6, no. 1-2, pp. 23-37, 1991.

[5] V. P. Simpson, "Optimum solution structure for a repairable inventory problem," Operations Research, vol. 26, no. 2, pp. 270281, 1978.

[6] K. Inderfurth, "Simple optimal replenishment and disposal policies for a product recovery system with leadtimes," Operations-Research-Spektrum, vol. 19, no. 2, pp. 111-122, 1997.

[7] S. X. Zhou, Z. T. Tao, and X. C. Chao, "Optimal control of inventory systems with multiple types of remanufacturable products," Manufacturing \& Service Operations Management, vol. 13, no. 1, pp. 20-34, 2011.

[8] Z. Tao, S. X. Zhou, and C. S. Tang, "Managing a remanufacturing system with random yield: properties, observations, and heuristics," Production and Operations Management, vol. 21, no. 5, pp. 797-813, 2012.

[9] G. A. DeCroix and P. H. Zipkin, "Inventory management for an assembly system with product or component returns," Management Science, vol. 51, no. 8, pp. 1250-1265, 2005.

[10] G. A. DeCroix, "Optimal policy for a multiechelon inventory system with remanufacturing," Operations Research, vol. 54, no. 3, pp. 532-543, 2006.

[11] D. P. Heyman, "Optimal disposal policies for a single-item inventory system with returns," Naval Research Logistics Quarterly, vol. 24, no. 3, pp. 385-405, 1977.

[12] J. A. Muckstadt and M. H. Isacc, "An analysis of single item inventory systems with returns," Naval Research Logistics Quarterly, vol. 28, no. 2, pp. 237-254, 1981.

[13] E. Van der Laan and M. Salomon, "Production planning and inventory control with remanufacturing and disposal," European Journal of Operational Research, vol. 102, no. 2, pp. 264278, 1997.

[14] E. Van der Laan, M. Salomon, R. Dekker, and L. Van Wassenhove, "Inventory control in hybrid systems with remanufacturing," Management Science, vol. 45, no. 5, pp. 733-747, 1999.

[15] G. A. DeCroix, J.-S. Song, and P. H. Zipkin, "Managing an assemble-to-order system with returns," Manufacturing \& Service Operations Management, vol. 11, no. 1, pp. 144-159, 2009.

[16] E. Kim, S. Saghafian, and M. P. Van Oyen, "Joint control of production, remanufacturing, and disposal activities in a hybrid manufacturing-remanufacturing system," European Journal of Operational Research, vol. 231, no. 2, pp. 337-348, 2013.

[17] S. A. Lippman, "Applying a new device in the optimization of exponential queueing systems," Operations Research, vol. 23, no. 4, pp. 687-710, 1975. 


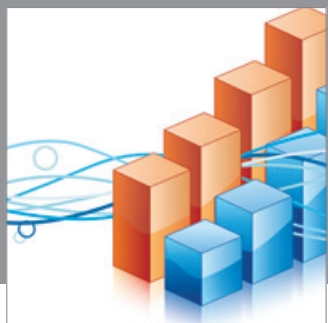

Advances in

Operations Research

mansans

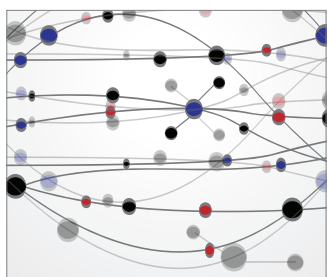

The Scientific World Journal
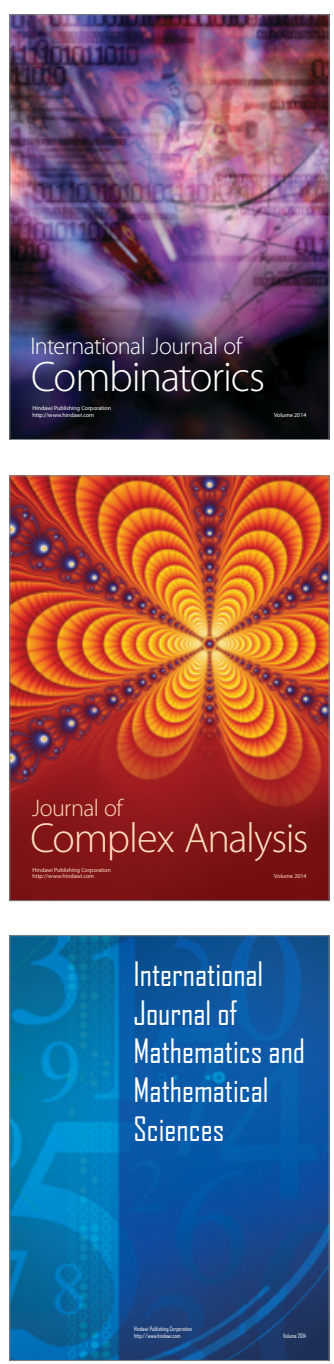
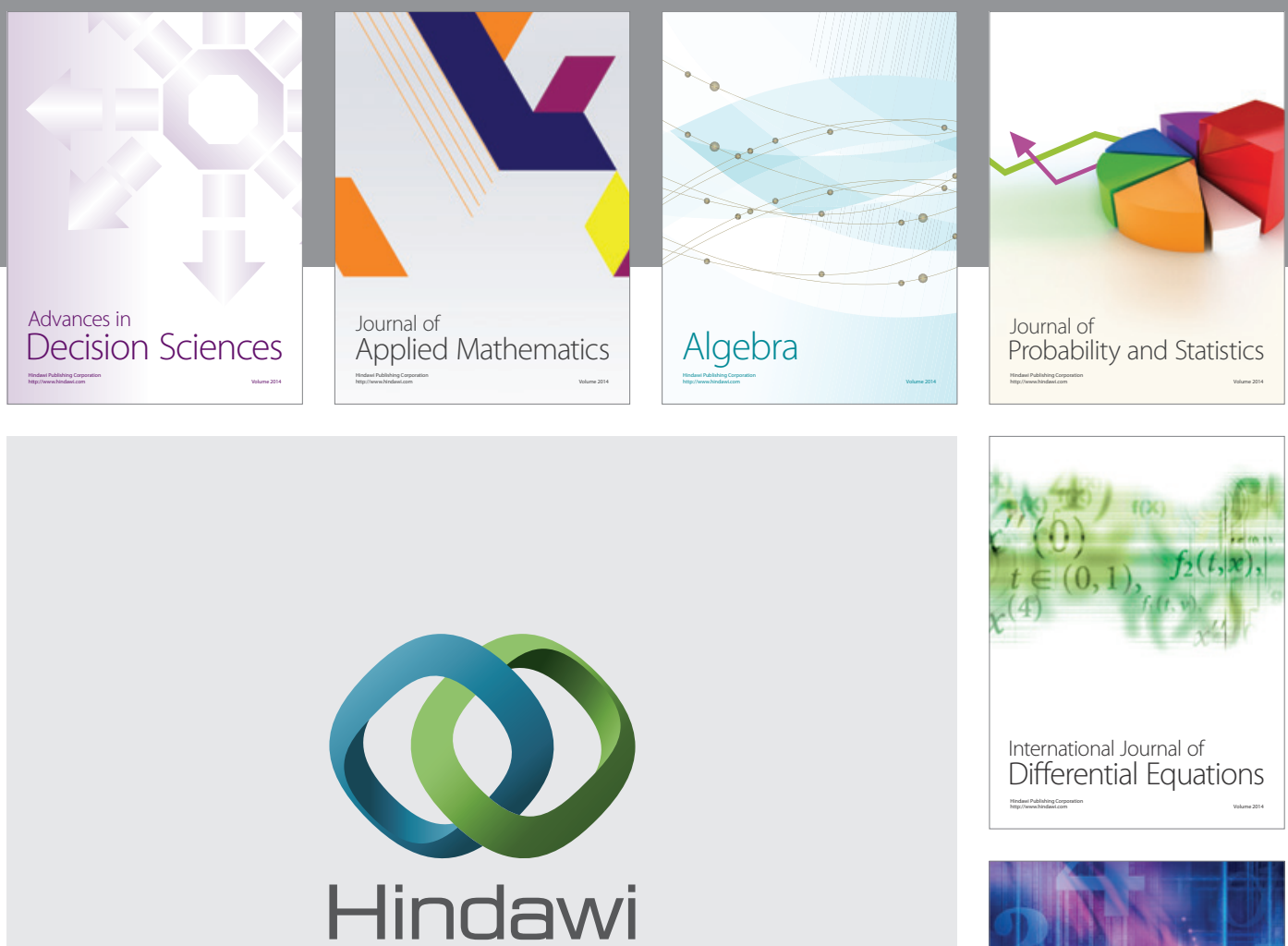

Submit your manuscripts at http://www.hindawi.com
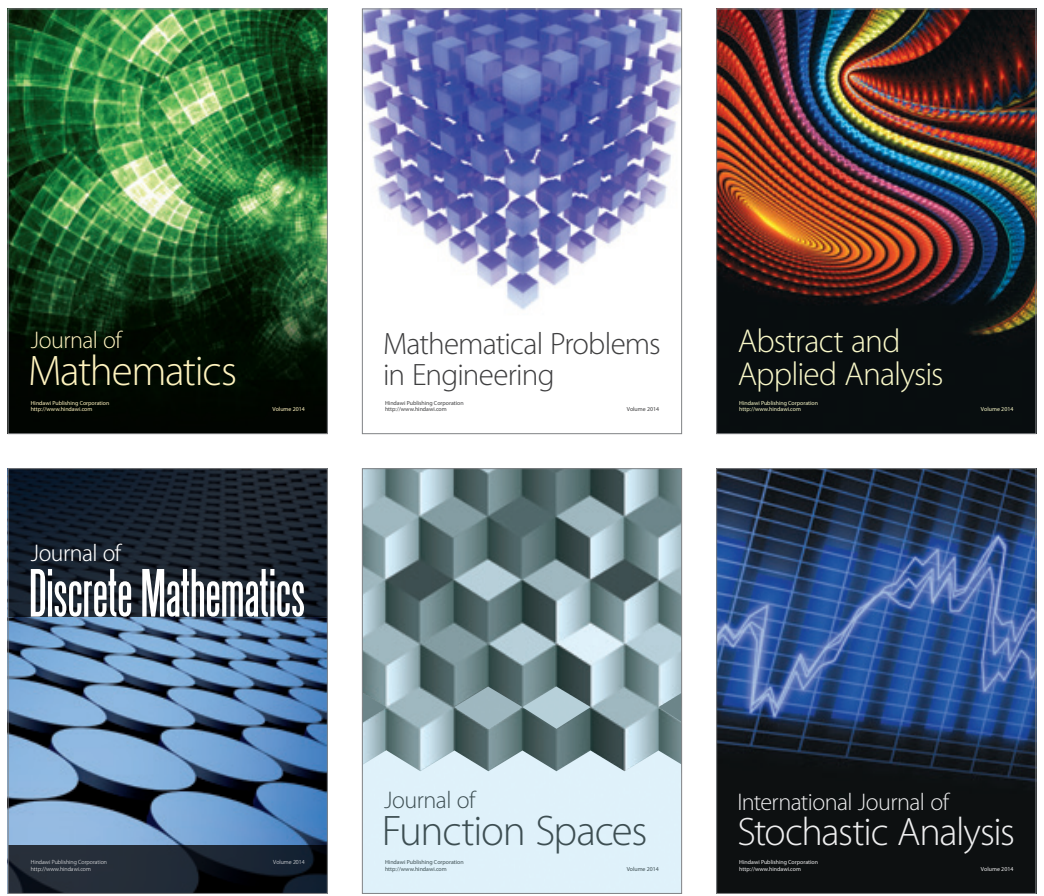

Journal of

Function Spaces

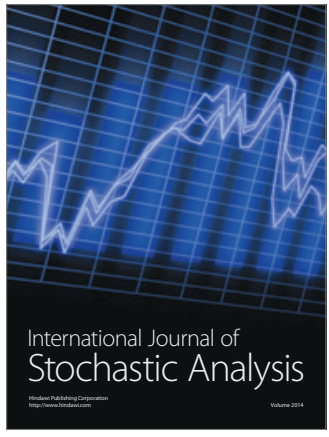

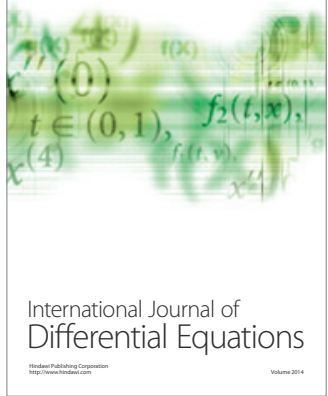
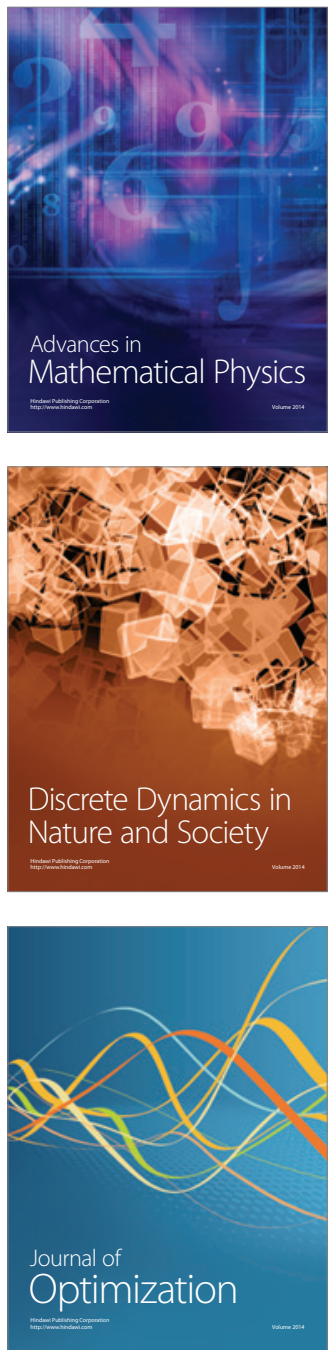